\title{
Padecer COVID-19 se asocia a un mayor riesgo para la salud a mediano plazo
}

\author{
Having COVID-19 is associated with an increased health risk in the medium term
}

\section{Comentado de:}

Al-Aly Z, et al. Nature. 2021 Apr 22. doi: 10.1038/s41586-02103553-9. Epub ahead of print. PMID: $33887749^{1}$

\section{Introducción}

Las manifestaciones clínicas agudas del COVID-19 están bien caracterizadas; sin embargo, sus secuelas luego de la fase aguda no han sido descritas en forma exhaustiva.

\section{Métodos}

Los autores utilizaron las bases de datos nacionales de atención médica del Departamento de Asuntos de Veteranos (VHA, por sus iniciales en inglés) de los EE.UU. para identificar de manera completa y sistemática las secuelas a seis meses (incluidos los diagnósticos, el uso de medicamentos y las anomalías de laboratorio), en sobrevivientes a la COVID-19, luego de 30 días de realizado el diagnóstico. Se utilizó un enfoque de caracterización de alta dimensión que comparó, en una cohorte de pacientes que no habían sido hospitalizados, 73.435 sobrevivientes de COVID19 y 4.990 .835 usuarios del VHA (medianas de seguimiento de 126 y 130 días, respectivamente).

También se comparó en una cohorte de pacientes que habían sido hospitalizados, a los 13.654 sobrevivientes de COVID-19 ( $26 \%$ de los cuales estuvieron en unidad de cuidados intensivos) con los 13.997 sobrevivientes de influenza estacional (medianas de seguimiento de 150 y 157 días, respectivamente). Para equilibrar la duración del seguimiento en ambos grupos de personas hospitalizadas, cada individuo del grupo ïnfluenza estacional"fue asignado al azar en forma independiente a una duración del seguimiento basada en la distribución de la duración del seguimiento de los participantes hospitalizados con COVID19 que habían sido seguidos desde la fecha de hospitalización hasta el 31 de enero de 2021.

Fueron evaluados 379 diagnósticos de enfermedades posiblemente relacionadas con COVID-19, 380 clases de medicamentos recetados y 62 pruebas de laboratorio administradas. Por otro lado, en ambas cohortes fueron analizados los resultados de desenlaces no relacionados con COVID-19, -como cánceres y accidentes, que deberían haber sido similares en los grupos con y $\sin$ COVID-19.

Las covariables predefinidas algorítmicamente para ajustar los análisis por posibles factores de confusión incluyeron variables demográficas como la edad, la raza, el sexo, la recepción de cuidados a largo plazo; indicadores de uso de recursos en salud como el número de consultas ambulatorias e internaciones, el número de recetas para pacientes ambulatorios, y el número de determinaciones de filtrado glomerular durante el año previo al diagnóstico; el nivel socioeconómico estimado por área de residencia, y la evaluación secuencial de insuficiencia orgánica (SOFA, por sus iniciales en inglés), como indicador de la gravedad de la enfermedad aguda.

El examen de las diferencias estandarizadas de todas las variables mencionadas y en todas las cohortes, mostró que más del $99,99 \%$ de las diferencias estandarizadas fueron $<0,1$ después del ajuste, confirmando una alta comparabilidad entre grupos.

\section{Resultados}

Los veteranos participantes de estudio fueron principalmente hombres. En la cohorte de no hospitalizados eran $88 \%$ y en la de hospitalizados $94 \%$, pero dado el gran tamaño de la muestra, aún incluyó a 8.880 y 793 mujeres con COVID-19 respectivamente.

Más allá de los primeros 30 días de enfermedad y luego de realizar el ajuste estadístico, las personas que tuvieron COVID-19 sin haber sido hospitalizadas exhibieron un mayor riesgo de muerte (hazard ratio [HR] 1,59; intervalo de confianza [IC] del 95\% 1,46 a $1,73)$ que los controles. Esto equivale a un exceso de muertes de $8,39(7,09$ a 9,58) cada 1.000 sobrevivientes luego de la estandarización. También se observaron más consultas ambulatorias (HR 1,20; IC $95 \% 1,19$ a 1,21).

Fueron identificadas secuelas (excesos que variaron de 3 a 30 cada 1.000 sobrevivientes) en la mayoría de órganos y sistemas, que incluyeron:

- Sistema respiratorio: tos persistente, dificultad para respirar e hipoxemia.

- Sistema nervioso: accidente cerebrovascular, cefalea, problemas de memoria y problemas con los sentidos del gusto y el olfato.

- Salud mental: ansiedad, depresión, problemas de sueño y abuso de sustancias.

- Metabolismo: nueva aparición de diabetes, obesidad y colesterol alto.

- Sistema cardiovascular: enfermedad coronaria aguda, insuficiencia cardíaca, palpitaciones y arritmias.

- Sistema gastrointestinal: constipación, diarrea y reflujo.

- Riñón: lesión renal aguda y enfermedad renal crónica que, ocasionalmente requirió diálisis.

- Coagulación: trombos en piernas y pulmones.

- Piel: erupción y caída del cabello.

- Sistema musculoesquelético: dolor articular y debilidad muscular.

- Salud general: malestar, fatiga y anemia.

Si bien ningún sobreviviente sufrió todos estos problemas, muchos desarrollaron un conjunto de varios problemas, con un considerable impacto en su salud y su calidad de vida.

Se observó un mayor uso de analgésicos (opioides y no opioides), antidepresivos, ansiolíticos, antihipertensivos e hipoglucemiantes orales y se evidenciaron múltiples anomalías de laboratorio. Los análisis preespecificados revelaron un gradiente de riesgo que aumentó según la gravedad de la infección aguda por COVID-19 (no hospitalizado, internación general, internación en cuidados intensivos) para los desenlaces clínicos, excluyendo el riesgo de muerte.

Cuando se comparó a los pacientes internados sobrevivientes de COVID-19 con los sobrevivientes de influenza estacional (aparentemente de la estación inmediata anterior), los resultados fueron similares. Los supervivientes de COVID-19 evidenciaron un mayor riesgo de muerte (HR 1,51; IC95\% 1,30, 1,76), equivalente a un exceso de muerte de 28,79 (IC95\% 19,52; 36,85) por 1000 personas a los seis meses.

También comparados con los pacientes hospitalizados por otras causas distintas a COVID-19 $(n=901.516)$ arrojó resultados consistentes.

Además, los resultados vinculados a desenlaces no relacionados con COVID-19 (cánceres y lesiones no intencionales) fueron similares en todos los grupos. Por otro lado y a diferencia de 
haber sufrido COVID-19, haber cursado influenza no se asoció de manera estadísticamente significativa con los mismos desenlaces.

\section{Conclusiones}

Los sobrevivientes de COVID-19, particularmente los pacientes más severos, experimentan una sustancial pérdida de salud, tanto respecto de su compromiso pulmonar como extrapulmonar e incluso en el riesgo de morir hasta los seis meses de haber padecido la enfermedad. Estos resultados permitirían la planificación de estrategias de atención multidisciplinaria en el sistema de salud para reducir la pérdida crónica de salud en estos pacientes.

\section{Comentario}

Las manifestaciones clínicas agudas de COVID-19 han sido bien estudiadas ${ }^{2}$, pero el presente estudio ${ }^{1}$ arroja luz sobre sus secuelas luego de la fase aguda.

Este riguroso estudio sugiere un mayor riesgo de muerte y permitió identificar el gran espectro de desenlaces clínicos y de uso de recursos experimentados en exceso por sobrevivientes de COVID-19 más allá de los 30 días de la enfermedad. Este estudio difiere de otros que han analizado COVID-19 a largo plazo, porque, en lugar de centrarse solo en las complicaciones neurológicas o cardiovasculares, adoptó una espectro muchísimo más amplio. Los análisis preespecificados de desenlaces no relacionados con COVID-19, las comparaciones con los pacientes con influenza y el gradiente severidad de la enfermedad o de los desenlaces, complementan y otorgan gran robustez a sus hallazgos, llevado a cabo en una extensa cohorte de pacientes. Este es el mayor estudio de COVID-19 post-agudo realizado a la fecha, que involucró millones de participantes y se basó en el mayor sistema de prestación de servicios de salud integrado a nivel nacional en los EE.UU.

Sin embargo, no está exento de limitaciones. Por un lado, no distingue las consecuencias directas de las indirectas del COVID-19. Por otro, la composición predominantemente masculina de la población en estudio no permite extrapolar sus conclusiones a la población femenina. Además, no deja de ser un estudio de bases de datos por lo que no se puede descartar completamente la confusión residual, especialmente teniendo en cuenta que de la descripción de los autores no surge con claridad cuál fue estructura de confusión teórica que se tomó como marco para el análisis causal.

Finalmente, como la pandemia de el COVID-19 sigue evolucionando y surgen nuevas variantes del virus, a la vez que mejores tratamientos y aumenta la disponibilidad de la vacuna, es probable que la epidemiología de esta enfermedad, a corto y largo plazo, también se modifique. Por otro lado, los mecanismos subyacentes a las manifestaciones post-agudas y crónicas de COVID-19 no están del todo claras. Algunas de las manifestaciones pudieron ser de origen inmunológico, pero también deben considerarse posibles efectos indirectos que incluyen los cambios sociales ( $p$. ej., menor contacto social y soledad), económicos (p. ej., pérdida de empleo) y en el comportamiento (p. ej., cambios en la dieta y el ejercicio) que pueden experimentar los sobrevivientes de COVID-19³.

\section{Conclusiones del comentador}

Los hallazgos muestran que más allá de los primeros 30 días de enfermedad, la COVID-19 impone una carga sustancial en salud, que abarca una amplio espectro de enfermedades pulmonares y extrapulmonares. La mayor severidad se asoció a mayor morbilidad, y también a un mayor riesgo de muerte, con excesos de muertes, por encima de los esperable, de 8 a 29 casos cada 1000 sobrevivientes en pacientes no hospitalizados y hospitalizados respectivamente. Considerando la enorme masa de pacientes recuperados, de casi 150 millones de personas ${ }^{4}$, el mundo debiera prepararse, al menos esta vez, para un nuevo tipo de ola de enfermedad post-COVID-19 de proporciones aún desconocidas.

Agustín Ciapponi [ Servicio de Medicina Familiar y Comunitaria, Hospital Italiano de Buenos Aires; Centro Cochrane Argentina, Instituto de Efectividad Clínica y Sanitaria. aciapponi@gmail.com ]

Ciapponi A. Padecer COVID-19 se asocia a un mayor riesgo para la salud a mediano plazo. Evid Actual Pract Ambul. 2021;24(2):e002126. Comentado de: Al-Aly Z, et al. High-dimensional characterization of post-acute sequalae of COVID-19. Nature. 2021 Apr 22. doi: 10.1038/s41586-021-03553-9. Epub ahead of print. PMID: 33887749

\section{Referencias}

1. Al-Aly Z, Xie Y, Bowe B. High-dimensional characterization of post-acute sequalae of COVID-19. Nature. 2021;Available from: 10.1038/s41586-02103553-9;https://dx.doi.org/10.1038/s41586-021-03553-9.

2. Xie Y, Bowe B, Maddukuri G, et al. Comparative evaluation of clinical manifestations and risk of death in patients admitted to hospital with covid-19 and seasonal influenza: cohort study. BMJ. 2020;371:m4677-m4677. Available from: 10.1136/bmj.m4677;https://dx.doi.org/10.1136/bmj.m4677.

3. Figueroa JD, Brennan PM, Theodoratou E. Distinguishing between direct and indirect consequences of covid-19. BMJ. 2020;369:2377-2377. Available from: $10.1136 / \mathrm{bmj} . \mathrm{m} 2377$

4. COVID-19 Data Repository by the Center for Systems Science and Engineering (CSSE) at Johns Hopkins University; 2021. Available from: https: //www.arcgis.com/apps/opsdashboard/index.html\#/bda7594740fd40299423467b48e9ecf6 [Last access: 2021-04-27]. 